\title{
Existence Conditions of High-k Modes in Finite Hyperbolic Metamaterials
}

Mahmoodi, Maryam; Tavassoli, Seyed Hassan; Takayama, Osamu; Sukham, Johneph; Malureanu, Radu; Lavrinenko, Andrei V.

Published in:

Laser \& Photonics Reviews

Link to article, DOI:

10.1002//por.201800253

Publication date:

2019

Document Version

Peer reviewed version

Link back to DTU Orbit

Citation (APA):

Mahmoodi, M., Tavassoli, S. H., Takayama, O., Sukham, J., Malureanu, R., \& Lavrinenko, A. V. (2019).

Existence Conditions of High-k Modes in Finite Hyperbolic Metamaterials. Laser \& Photonics Reviews, 13(3), [1800253]. https://doi.org/10.1002//por.201800253

\section{General rights}

Copyright and moral rights for the publications made accessible in the public portal are retained by the authors and/or other copyright owners and it is a condition of accessing publications that users recognise and abide by the legal requirements associated with these rights.

- Users may download and print one copy of any publication from the public portal for the purpose of private study or research.

- You may not further distribute the material or use it for any profit-making activity or commercial gain

- You may freely distribute the URL identifying the publication in the public portal 
Abstract The capability to support optical waves with very large wave vectors (high-k) is one of the principle features of hyperbolic metamaterials (HMMs). These waves play the key role in HMM applications such as imaging and lifetime engineering. Effective medium approximation (EMA) as widely used analytical method to predict HMMs behavior, has shortcomings in calculating high$\mathrm{k}$ modes of practical structures. EMA is applicable to a subwavelength unit-cell of implicitly infinite periodic structures. Using conventional EMA, in the present paper, boundary effects and spatial dispersion are taken into consideration to properly compute the high-k modes of finite-thickness multilayer HMMs. Applying nonlocal homogenization to stacks of alternating metal-dielectric layers, the corresponding effective medium is examined as a high-k waveguide sandwiched between the substrate and an ambient superstrate. The developed theory enables us to recognize two types of bulk waves coined as shortrange and long-range propagating modes. Number of such modes as well as their cut-off conditions are quantified for the first time. Validity of the developed theory is verified both numerically by rigorous simulations of the multilayer structures with the transfer matrix method and experimentally by optical characterization of the HMMs in infrared regime.

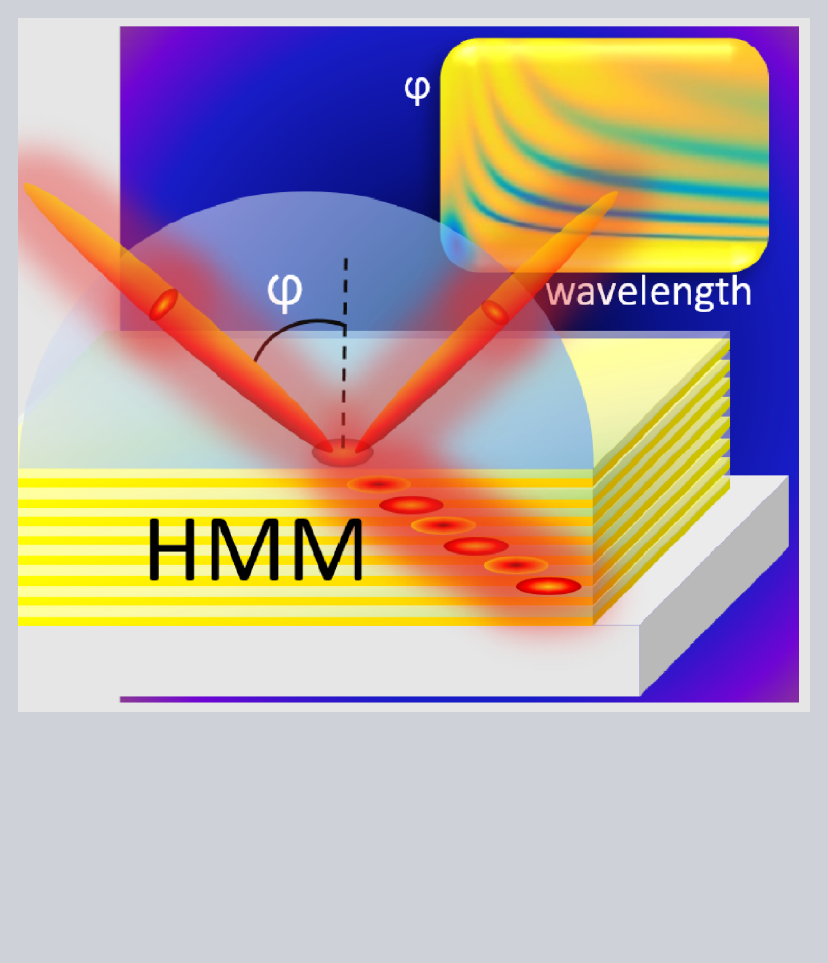

\title{
Existence conditions of high-k modes in finite hyperbolic
} metamaterials

\author{
Maryam Mahmoodi ${ }^{1}$, Seyed Hassan Tavassoli ${ }^{1,}$, Osamu Takayama ${ }^{2}$,Johneph Sukham ${ }^{2}$, Radu \\ Malureanu ${ }^{2}$ and Andrei V. Lavrinenko ${ }^{2}$
}

\section{Introduction}

Artificial materials with unique properties are approaching toward practical implementation as material science and nano-fabrication technologies are rapidly developing. Artificially engineered structures with distinctive electromagnetic and optical properties known as metamaterials, [1] have been standing out of top research interests in the last decade. Among them stands the promising class of hyperbolic metamaterials (HMMs), [2,3] with applications such as sub-diffraction imaging, [4-6] sensing, $[7,8]$ nanoscale manipulation of light-matter interaction like directional propagation of light, [9-13] and lifetime engineering. [14-19] Subwavelength metal-dielectric multilayer HMMs [20,21] amongst the other configurations such as nanotrenches $[8,13]$ and nanowires, $[7,22]$ have attracted widespread interests due to relatively ease of fabrication and extended nomenclature of materials enabling the hyperbolic regime over a broad range of frequencies.

It is ascertained that the open form (hyperboloidshaped) of HMMs iso-frequency surface leads to optical modes with high effective mode index and respectively high wave vectors. In spite of the vital role of these high-k bulk Bloch modes in HMMs applications, [23] a comprehensive theory predicting their behavior in real case (finite-size structures) is still missing. Bloch theory is the principal approach to investigate infinite periodic composites via their band structures. Yet, it is unable to explicate appearance and existing conditions for the high-k modes in multilayer structures. Homogenization theories applied to a subwavelength unit cell of infinite periodic multilayers, [24] can be used to describe the composites with uniform effective media rather than band structures. However, the effective medium approximation (EMA)

${ }^{1}$ Laser and Plasma Research Institute, Shahid Beheshti University, Tehran, Iran ${ }^{2}$ Technical University of Denmark, Dept. of Photonics Engineering, Ørsteds plads, bldg. 345V, 2800 Kgs. Lyngby, Denmark

* Corresponding author: e-mail: h-tavassoli@sbu.ac.ir 
being vastly used in literature, [25] also shows major flaws in predicting behaviour of HMMs in terms of supporting high-k modes. EMA is generally applied to one unit cell of periodic structures and cannot discriminate properties of effective media obtained from stacks having different number of periods. Independence of EMA from number of periods (or alternatively from the HMM thickness) has raised challenges to correctly assert effective medium properties out of a real multilayer stack with finite thickness. On the other hand, necessity for the unit cell of periodic structures to be deeply subwavelength poses notable nonlocality effects not considered in the conventional EMA. Consequently, it fails to correctly calculate the high-k modes and provide adequate proof for their effective mode index cut-offs reported in many experimental and numerical studies. [26-29] Meanwhile, although straight numerical calculations, such as the well-known transfer matrix method (TMM), $[30,31]$ deliver practically exact solutions, they suffer from ambiguity in explaining nature of the modes. Therefore, it leaves a great demand for a simple theory that deliver high-k modes properties in practical HMMs.

In the present paper we developed a theory based on the effective medium approximation to overcome notorious limitations of conventional EMA, nonlocality $[32-34]$ and boundary effects. [35] To probe the high-k modes of finite multilayer HMMs, the nonlocal homogenization procedure is applied. The corresponding effective medium is considered as a waveguide-like slab with a finite thickness supporting propagation of high-k modes. Consequently, two kinds of bulk propagating modes in the HMM slab are distinguished and named as short-range and long-range propagating modes. The developed dispersion relations properly explain the cut-off conditions and number of modes in any arbitrary multilayer HMMs. Transfer matrix method and experimental characterization of HMMs consisting of gold-alumina multilayers ascertain the results of developed theory.

\section{Infinite HMMs: Nonlocal Homogenization}

By definition, hyperbolic metamaterials are identified as anisotropic media possessing hyperbolic isofrequency surface for TM-polarized waves. [36] This definition denotes the effective medium approximated from the sub-wavelength structures. The conventional homogenization can be done by the well-known Maxwell Garnett approach. Considering multilayer structures composed of periodic nonmagnetic metal-dielectric layers with permittivities $\varepsilon_{\mathrm{m}}, \varepsilon_{\mathrm{d}}$ and thickness $t_{\mathrm{m}}, t_{\mathrm{d}}$ $(<<\lambda)$ respectively, the local EMA predicts a uniaxial permittivity tensor for the effective medium with the following elements.

$$
\begin{aligned}
& \varepsilon_{\mathrm{o}}=p \varepsilon_{\mathrm{m}}+(1-p) \varepsilon_{\mathrm{d}} \\
& \varepsilon_{\mathrm{e}}=\left(\frac{p}{\varepsilon_{\mathrm{m}}}+\frac{1-p}{\varepsilon_{\mathrm{d}}}\right)^{-1}
\end{aligned}
$$

where $\lambda$ is the vacuum wavelength and $p$ is the metal fill fraction in the unit cell of $t=t_{\mathrm{m}}+t_{\mathrm{d}}$. The extraordinary $\varepsilon_{\mathrm{e}}$ and ordinary $\varepsilon_{\mathrm{o}}$ permittivities must satisfy the condition $\varepsilon_{\mathrm{e}} \cdot \varepsilon_{\mathrm{o}}<0$ in order for the effective medium to be in the hyperbolic regime. For working frequen-

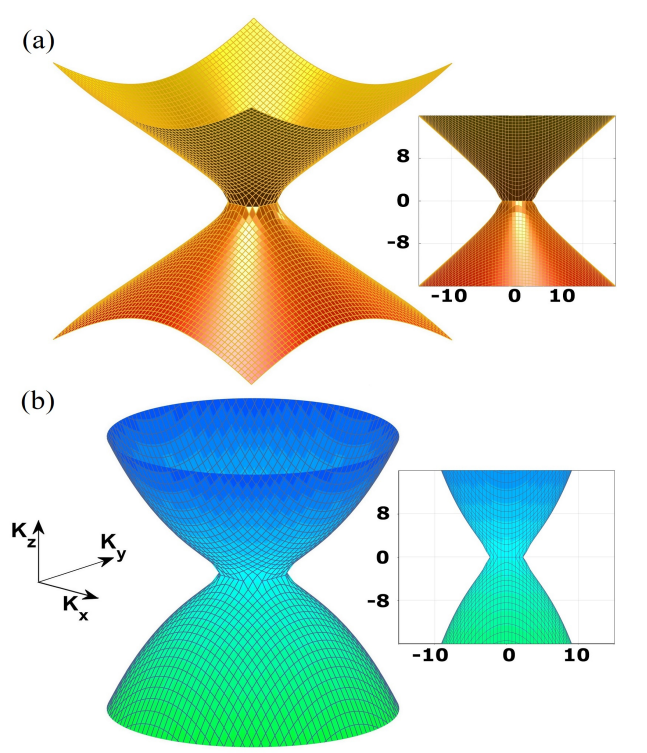

Figure 1 Isofrequency surface of type II hyperbolic metamaterials, implementing a) local b) nonlocal effective medium approximation.

cies in visible-IR, metal-dielectric stacks are typically characterized as type II HMMs with positive $\varepsilon_{\mathrm{e}}$ and negative $\varepsilon_{0}$. [28] Recently, the local homogenization of subwavelength multilayer structures was shown to be inadequate to accurately calculate the effective properties. [37-40] To resolve the limitation, homogenization procedure must become nonlocal by taking dependency of permittivities to wavevector into consideration. To account for nonlocality, we utilized operator approach effective medium theory where the components of permittivity tensor can be represented in a power series of $k_{0} t$, with $k_{0}$ being the vacuum wavenumber. [41] For periodic multilayer structures with a bilayer unit cell, as common and most used one, the nonlocal permittivity components written up to $\left(k_{0} t\right)^{2}$ can be defined as

$$
\begin{gathered}
\varepsilon_{\mathrm{o}}^{\mathrm{nl}}=\varepsilon_{\mathrm{o}}+\frac{\left(k_{0} t\right)^{2}}{6} \sigma \varepsilon_{\|} f(\beta) \\
\varepsilon_{\mathrm{e}}^{\mathrm{nl}}=\left\{\frac{1}{\varepsilon_{\mathrm{e}}}+\frac{\left(k_{0} t\right)^{2}}{6} \sigma\left(\frac{2 p-1}{\varepsilon_{\mathrm{r}}}-\frac{f(\beta)}{\varepsilon_{\perp}}\right)\right\}^{-1}
\end{gathered}
$$


Where followings are parameters related to the unitcell feature and $\beta$ is the normalized transverse wavevector. Henceforth we identify $\beta$ as effective index.

$$
\begin{aligned}
\varepsilon_{\|} & =p \varepsilon_{\mathrm{m}}-(1-p) \varepsilon_{\mathrm{d}}, \\
\varepsilon_{\perp} & =\left(\frac{p}{\varepsilon_{\mathrm{m}}}-\frac{1-p}{\varepsilon_{\mathrm{d}}}\right)^{-1} \\
\varepsilon_{\mathrm{r}} & =\left(\frac{1}{\varepsilon_{\mathrm{m}}}+\frac{1}{\varepsilon_{\mathrm{d}}}\right)^{-1}, \\
\sigma & =p(1-p)\left(\varepsilon_{\mathrm{d}}-\varepsilon_{\mathrm{m}}\right), \\
f(\beta) & =\frac{\beta^{2}}{\varepsilon_{\mathrm{r}}}-1
\end{aligned}
$$

The well-known dispersion equation of bulk high-k waves of the HMMs is given as [28]

$$
\frac{k_{\mathrm{x}}^{2}+k_{\mathrm{y}}^{2}}{\varepsilon_{\mathrm{e}}}+\frac{k_{\mathrm{z}}^{2}}{\varepsilon_{\mathrm{o}}}=k_{0}^{2}
$$

where the coordinate system is chosen such as its z-axis coincides with the optical axis (being normal to the layer interfaces). To display the above dispersion relation, a typical subwavelength multilayer structure consisting of gold and alumina layers is considered. Figure 1a represents the hyperboloidal isofrequency surface of the correspondng type II hyperbolic metamaterial. By replacing local with nonlocal parameters in Eq. (3), the isofrequency surface in nonlocal regime is depicted in Figure 1b. The $2 \mathrm{D}$ views, shown in the same scale, are beneficial for better comparison. Figure 1 visualizes the significant role of nonlocality in dispersion of the high-k waves and associated wave vectors.

Following from Eq. (3) and utilizing nonlocal parameters, vertical component of the wavevector, $k_{\mathrm{z}}$, will be as

$$
k_{\mathrm{z}}=k_{0} \sqrt{-\varepsilon_{\mathrm{o}}^{\mathrm{nl}}\left(\frac{\beta^{2}}{\varepsilon_{\mathrm{e}}^{\mathrm{nl}}}-1\right)}
$$

Note that due to symmetry in $\mathrm{x}$ and $\mathrm{y}$ axes, without loss of generality, the wave vector $\mathrm{k}$ is taken as $\mathrm{k}=\beta k_{0} \hat{\mathrm{x}}+k_{\mathrm{z}} \hat{\mathrm{z}}$ and $\beta=\frac{k_{\mathrm{x}}}{k_{0}}$. Since the permittivity parameters are complex values, $k_{\mathrm{z}}$ is generally a complex parameter. Dependency of nonlocal parameters of permittivity to the effective index, makes a complicated relation between $k_{\mathrm{z}}$ and $\beta$. However, for type II HMMs with $\varepsilon_{\mathrm{o}}^{\mathrm{nl}}<0$ and $\varepsilon_{\mathrm{e}}^{\mathrm{nl}}>0$, we can analytically explore two plausible scenarios for propagation of bulk high-k waves inside the HMM structures;

\section{Long-Range (LR) high-k waves}

Considering the inequality $\varepsilon_{\mathrm{e}}^{\mathrm{nl}}<\beta^{2}$, the expression under above square root would be a positive value and provides $k_{\mathrm{z}}$ with a major real part. Consequently, the waves will be propagating along the $\mathrm{z}$ axis with a minor damping caused by the imaginary part. We coined the waves of this category as Long-Range (LR) propagating high-k waves of HMMs.

\section{Short-Range (SR) high-k waves}

On the contrary, if $\varepsilon_{\mathrm{e}}^{\mathrm{nl}}>\beta^{2}$ it results in a large imaginary part for $k_{\mathrm{z}}$. Under this condition, bulk high-k waves propagate in the HMM within a short propagation length and undergo a major decay. These high damping bulk waves are conceived and coined as ShortRange (SR) propagating high-k waves of HMMs.

Summing up our considerations, nonlocality results in two mutable categories of propagating high-k waves in multilayer HMMs. Variation in extraordinary permittivity $\sqrt{\varepsilon_{\mathrm{e}}^{\mathrm{nl}}(\beta)}$ by changing effective index, rules short or long range propagation of the HMMs high-k waves. We would like to mention that our theory takes into consideration the nonlocality effect arising from geometry of the layers, but not from the materials properties. In order to account for deviation of permittivity of the materials involved from standard descriptions, such as nonlocality of very thin metallic films, [42] one should use the corrected expressions of $\varepsilon_{\mathrm{m}}$ and $\varepsilon_{\mathrm{d}}$ in the above equations. However, such case is beyond the scope of this article.

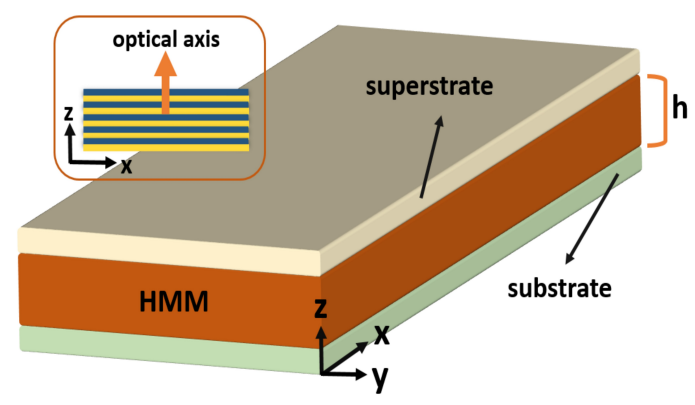

Figure 2 Finite-thickness HMMs represented as asymmetric waveguides for HMMs high-k waves.

\section{Finite HMMs; Waveguide-like HMM slabs}

The simplest configuration to deliberate a finite thickness HMM would be a slab of effective medium sandwiched between two semi-infinite media. Following discussions in the previous section, HMMs generally support short-range and long-range propagating waves having large wave vectors that cannot exist outside the HMMs. Thus, a finite thickness HMM looks like a waveguide for these high- $\mathrm{k}$ waves by keeping them confined within the HMM core. Figure 2 schematically shows the finite thickness HMMs in such waveguide configuration. In general, an asymmetric waveguide consisting of the HMM slab as core and dielectrics with permittivities $\varepsilon_{1}$ and $\varepsilon_{2}$ as cladding is considered, taking $\varepsilon_{1} \leqslant \varepsilon_{2}$ for simplicity. Within the framework of conventional optical slab waveguides, the effective refractive index of the middle layer must be greater than those of the surrounding media to satisfy confinement 
condition. In our case, this condition $\sqrt{\varepsilon_{1}} \leqslant \sqrt{\varepsilon_{2}}<\beta$ is automatically satisfied, since HMM high-k waves possess large effective index $\beta$ that makes them evanescent outside the core.

Naturally hyperbolic material [43] and multilayer HMMs [44-46] are recently studied as alternative configurations of plasmonic waveguides, looking for confined waves to the interfaces. Seeking for a general scheme, the present work investigates all kinds of possible bulk high-k waves in the HMM core; those propagating in the HMM slab over a long length and the ones more confined to the interface of HMM and soroundings. Therefore, considering the general rules and conditions of conventional waveguides, we continue our theoretical discussion to find dispersion relations of the SR and LR propagating modes in finite thickness HMMs.

\subsection{Theoretical model}

Considering three segments shown in Figure 2 as homogeneous media, electromagnetic handling of the problem is relatively straightforward. Solution of Maxwell's equation in homogeneous media are plane waves. [30] $\mathrm{TM}$ and TE polarization in the surrounding dielectrics and ordinary and extraordinary modes in the HMM slab, are normal modes of the media. [47] Since the optical axis lies in $\mathrm{z}$ direction, the two modes are separable in terms of polarization, extraordinary corresponds to TM and ordinary corresponds to TE polarization. Starting with Maxwell's equations, we investigate propagation of monochromatic TM-polarized plane waves along the z-axis in an anisotropic medium. Thus, $E_{\mathrm{x}}, E_{\mathrm{z}}$ and $H_{\mathrm{y}}$ of field components remain nonzero. Considering changes along the $\mathrm{x}$-axis as $\frac{\partial}{\partial x}=i k_{\mathrm{x}}$ and no field changes in y-axis, i.e. $\frac{\partial}{\partial y}=0$, the electric field amplitudes would be

$$
E_{\mathrm{z}}=\frac{-k_{\mathrm{x}}}{\omega \varepsilon_{\mathrm{z}}} H_{\mathrm{y}} \quad E_{\mathrm{x}}=\frac{-i}{\omega \varepsilon_{\mathrm{x}}} \frac{\partial}{\partial \mathrm{z}} H_{\mathrm{y}}
$$

Using $H$ simply instead of $H_{\mathrm{y}}$, one obtains the following eigenvalue expression with the parameter $\alpha=\frac{\varepsilon_{\mathrm{x}}}{\varepsilon_{\mathrm{z}}}$.

$$
\frac{\partial^{2} H}{\partial z^{2}}+\alpha\left(-k_{\mathrm{x}}^{2}+\varepsilon_{\mathrm{z}} \mu_{0} \omega^{2}\right) H=0
$$

For the HMM slab, $\alpha$ is a negative value and for the isotropic dielectrics, it is equal to unity. Applying the latter equation separately in each homogeneous segment of the waveguide, we conclude to the following equations for evanescent waves in the surrounding dielectric media and for evanescent and propagating waves in the HMM slab.

$$
\frac{\partial^{2} H}{\partial z^{2}}-k_{0}^{2}\left(\beta^{2}-\varepsilon_{1,2}\right) H=0
$$

$$
\begin{aligned}
& \frac{\partial^{2} H}{\partial z^{2}}-k_{0}^{2}\left(-\varepsilon_{\mathrm{o}}^{\mathrm{nl}}\left(1-\frac{\beta^{2}}{\varepsilon_{\mathrm{e}^{\mathrm{nl}}}}\right)\right) H=0 \\
& \frac{\partial^{2} H}{\partial z^{2}}+k_{0}^{2}\left(-\varepsilon_{\mathrm{o}}^{\mathrm{nl}}\left(\frac{\beta^{2}}{\varepsilon_{\mathrm{e}^{\mathrm{nl}}}}-1\right)\right) H=0
\end{aligned}
$$

To note, $\beta$ is conserved in all segments. Trial solutions for the $\mathrm{H}$ fields of evanescent waves in dielectrics, SR and LR high-k waves in the HMM slab are taken respectively as

$$
\begin{aligned}
& H_{1,2}(x, z, t)=H_{1,2}(z) \mathrm{e}^{i\left(k_{0} \beta x-\omega t\right)} \\
& H_{S R}(x, z, t)=H_{S R}(z) \mathrm{e}^{i\left(k_{0} \beta x-\omega t\right)} \\
& H_{L R}(x, z, t)=H_{L R}^{\mathrm{m}}(z) \mathrm{e}^{i\left(k_{0} \beta x-\omega t\right)}
\end{aligned}
$$

Here, $H_{L R}^{\mathrm{m}}(z)$ is the wave function of the LR high-k waves in $\mathrm{z}$ direction and $\mathrm{m}=1,2, \ldots$ refers to the mode number. Now, following the standard routine with continuity of $E_{\mathrm{x}}, E_{\mathrm{z}}$ and $\mathrm{H}$ across the interface of the HMM slab and applying Eq. (5) to Eq. (8) and using Eq. (7), one can derive dispersion relations of SR and LR high$\mathrm{k}$ modes as

$$
\begin{aligned}
& \tanh \left(\mathbb{K}_{\mathrm{SR}} k_{0} h\right)=\frac{\mathbb{K}_{\mathrm{SR}}\left(\varepsilon_{1} \mathbb{K}_{2}+\varepsilon_{2} \mathbb{K}_{1}\right)}{\frac{\varepsilon_{1} \varepsilon_{2}}{-\varepsilon_{\mathrm{o}}^{\mathrm{n}}} \mathbb{K}_{\mathrm{SR}}^{2}-\varepsilon_{\mathrm{o}}^{\mathrm{nl}} \mathbb{K}_{1} \mathbb{K}_{2}} \\
& \tan \left(\mathbb{K}_{\mathrm{LR}} k_{0} h\right)=\frac{\mathbb{K}_{\mathrm{LR}}\left(\varepsilon_{1} \mathbb{K}_{2}+\varepsilon_{2} \mathbb{K}_{1}\right)}{\frac{\varepsilon_{1} \varepsilon_{2}}{\varepsilon_{\mathrm{o}}^{\mathrm{nl}}} \mathbb{K}_{\mathrm{LR}}^{2}-\varepsilon_{\mathrm{o}}^{\mathrm{nl}} \mathbb{K}_{1} \mathbb{K}_{2}}
\end{aligned}
$$

where $\mathrm{h}$ stands for thickness of the HMM slab and $\mathbb{K}_{1}=\sqrt{\beta^{2}-\varepsilon_{1}}$ and $\mathbb{K}_{2}=\sqrt{\beta^{2}-\varepsilon_{2}}$ are the normalized vertical wavevectors of evanescent waves in surrounding media, $\mathbb{K}_{\mathrm{SR}}=\sqrt{-\varepsilon_{\mathrm{o}}^{\mathrm{nl}}\left(1-\frac{\beta^{2}}{\varepsilon_{\mathrm{e} \mathrm{nl}}}\right)}$ and $\mathbb{K}_{\mathrm{LR}}=\sqrt{-\varepsilon_{\mathrm{o}}^{\mathrm{nl}}\left(\frac{\beta^{2}}{\varepsilon_{\mathrm{e}} \mathrm{nl}}-1\right)}$ are those of SR and LR high-k waves in the HMM slab respectively. The above relations indicate that the bulk high-k modes dispersion of the HMM slab is strongly dependent on surrounding media as well as thickness of the effective medium $h$, which in turn is defined by the number of periods $\mathrm{N}$, and thickness of the unit cell $\mathrm{t}$, as $h=N t$. Solving the dispersion equations, the values of effective index $\beta$ for allowed bulk high-k modes in HMM slabs can be determined.

Figure 3 shows the high-k modes dispersion of multilayer stacks composed of $10 \mathrm{~nm}$-thick $\mathrm{Au}$ and $\mathrm{Al}_{2} \mathrm{O}_{3}$ in alternating order having a unit-cell of $\mathrm{p}=0.5$. It should be pointed out that metal's thickness correction to the Drude--Lorentz model, [48] is implemented in calculation of $\varepsilon_{\mathrm{m}}$. The corresponding HMM (effective medium) is sandwiched between a glass substrate $\left(n_{2}\right.$ $=1.51)$ and air as the superstrate $\left(n_{1}=1\right)$. The black lines show the SR modes; blue and red lines indicate the odd and even LR high-k modes in the HMM slabs. 


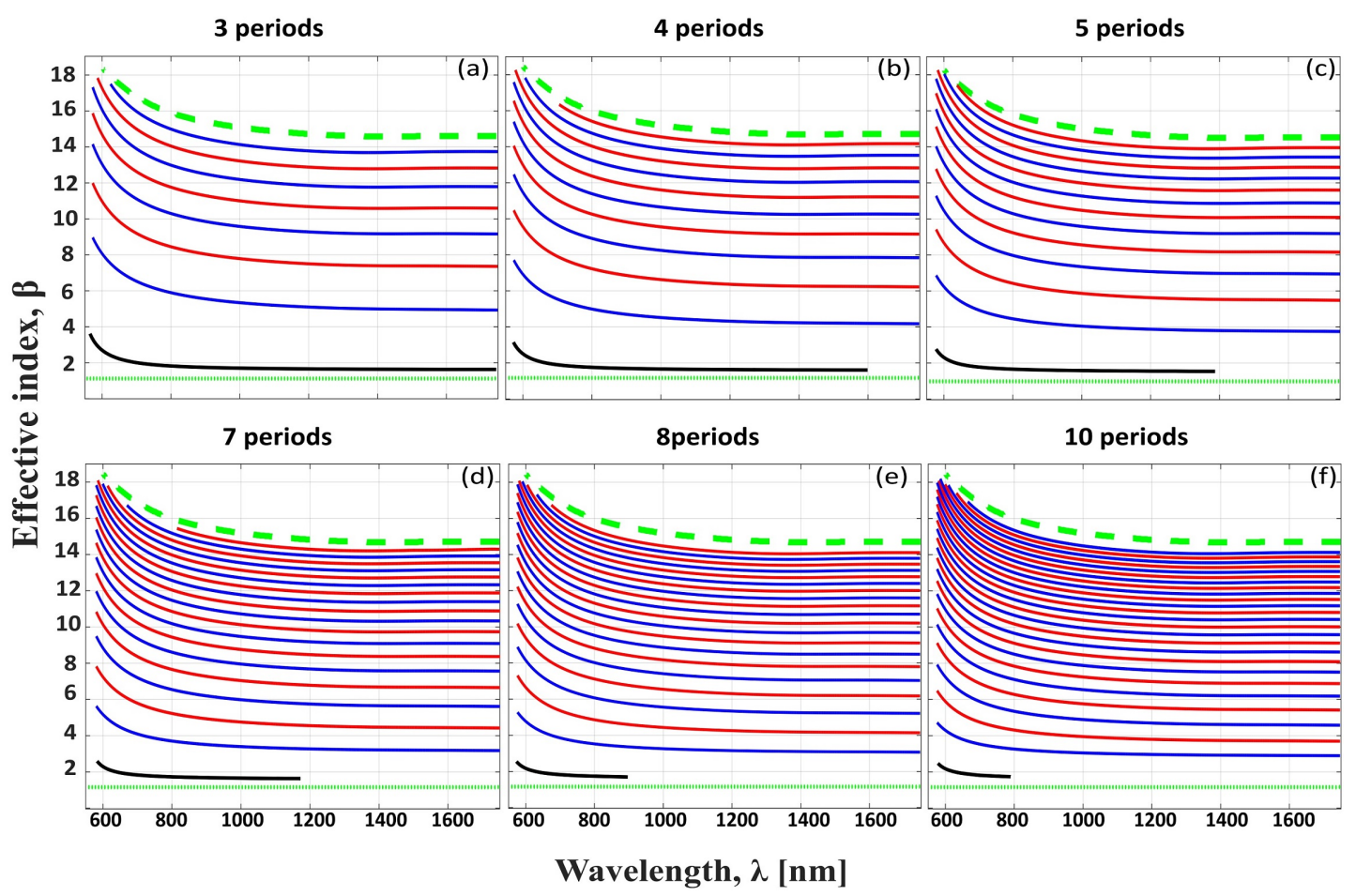

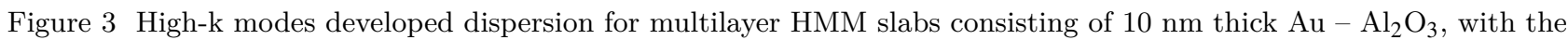
total thicknesses corresponding to a) 3, b) 4, c) 5, d) 7, e) 8, and f) 10 periods, sandwiched between glass substrate and air superstrate. Black lines show the short-range high-k modes, blue and red lines indicate the odd and even long-range high-k modes, respectively. The high-k modes lower and upper cut-offs are shown with the green dotted (lower) and dashed (upper) lines.

We exemplify cases with the HMM thickness of 60,80 , 100, 140, 160 and $200 \mathrm{~nm}$ equivalent to $3,4,5,7,8$, and 10 periods. The SR high-k modes appear for thin HMM slabs; as slabs thickness increases with an increased number of periods, short-range modes tend to fade as broadband waves. On the contrary, long-range high-k modes appear in HMM slabs with considerable thickness. By increasing thickness of the slab number of broadband LR modes increases and the corresponding effective mode index shifts to lower $\beta$.

\subsection{Cut-off Conditions}

As mentioned before, simulation and experimental studies of multilayer HMMs have shown that there exists a minimum as well as a maximum value for effective index of high-k modes of the structures. Neither minimum nor maximum values can be explained by applying conventional EMA. Due to the constraints imposed through boundaries and nonlocality, the developed theory provides us with the possibility of prediction and calculation of the limited span of possible $\beta$ for the effective mode indices of high-k waves.

In terms of mode confinement, a cut-off is reached when the modes begin to radiate outside the HMMcore waveguide so that the condition of high-k modes of the HMM slab is no longer satisfied. Following from section 2 , relation between the effective index $\beta$ and extraordinary permittivity, $\varepsilon_{\mathrm{e}}^{\text {nl }}$ of the HMM slab is a quite important parameter which determines how fast the bulk modes decay inside the HMM slab. The mentioned conditions together with the waveguide general constraints will determine the possible effective mode indices for the SR and LR modes.

$$
\begin{aligned}
& \varepsilon_{1} \leqslant \varepsilon_{2}<\beta^{2}<\varepsilon_{\mathrm{e}}^{\mathrm{nl}} \\
& \varepsilon_{1} \leqslant \varepsilon_{2}<\varepsilon_{\mathrm{e}}^{\mathrm{nl}}<\beta^{2}
\end{aligned}
$$

For short range modes, $\beta$ should satisfy the relation mentioned in Eq. (10a) and for the long range modes Eq. (10b) must be satisfied. Thus, two cut-offs can be recognized there. The lower cut-off, $\beta_{\text {lcf }}=\sqrt{\varepsilon_{2}}$, denotes the smallest possible effective mode index for the bulk modes. However, the upper cut-off is absolutely defined by the imposed nonlocality. Based on the nonlocal homogenization, $\varepsilon_{\mathrm{e}}^{\mathrm{nl}}$ is the function of effective index $\beta$. The bigger the $\beta$ is, the smaller gets the $\varepsilon_{\mathrm{e}}^{\mathrm{nl}}$. From Eq. (10a) one can conclude that SR modes exist with the effective mode indices spanning from $\beta_{\text {lcf }}$ to $\sqrt{\varepsilon_{\mathrm{e}}^{\mathrm{nl}}}$. As $\beta$ gets bigger, long range modes start to appear. Equation(10)b implies that $\beta$ attains its maximum value when $\varepsilon_{\mathrm{e}}^{\mathrm{nl}}$ reaches its minimum possible value $\varepsilon_{2}$. Consequently, the upper cut-off for bulk high-k modes can be found through imposing $\varepsilon_{\mathrm{e}}^{\mathrm{nl}} \approx \varepsilon_{2}$ 
constraint. Applying this condition to Eq. (2b) and defining $\varepsilon_{\mathrm{wg}}$ as the waveguide permittivity,

$$
\frac{1}{\varepsilon_{\mathrm{wg}}}=\frac{1}{\varepsilon_{2}}-\frac{1}{\varepsilon_{e}}
$$

one concludes to the following expression for LR modes upper cut-off, $\beta_{\text {ucf }}$.

$$
\beta_{\mathrm{ucf}}=\sqrt{\varepsilon_{\mathrm{r}}+\varepsilon_{\perp}(2 p-1)-\frac{\varepsilon_{\mathrm{r}} \varepsilon_{\perp}}{\varepsilon_{\mathrm{wg}}} \frac{3}{2 \pi^{2} \sigma}\left(\frac{\lambda}{t}\right)^{2}}
$$

It makes evident that the upper cut-off is related to the refractive index of the denser cladding as well as to the operating wavelength in addition to unit cell features. In Figure 3 the high-k modes cut-offs are shown with the green dotted (lower) and dashed (upper) lines. Figure 3 signifies that number of LR modes goes up as thickness of the HMM slab increases, yet the high$\mathrm{k}$ modes themselves are enclosed by the cut-off conditions which are independent from the HMM thickness.

\subsection{Number of LR modes}

Shown in Figure 3, number of LR modes grows up as thickness of the HMM slab increases. However, as a fact, number of high-k modes must be finite. At the point where $\beta$ reaches the maximum possible effective mode index, condition for existence of the high-k waves inside the HMM slab is not satisfied anymore and the wave is said to be cut-off. Writing the LR dispersion relation, Eq. (9a), at $\beta=\beta_{\text {ucf }}$ and considering the upper cut-off parameter $U^{\text {ucf }}$ as

$$
\begin{aligned}
& U^{\mathrm{ucf}}= \\
& \tan ^{-1}\left(\frac{\sqrt{-\varepsilon_{0}^{\mathrm{nl}}\left(\frac{\beta_{\mathrm{ucf}}^{2}}{\varepsilon_{\mathrm{e}}^{\mathrm{n}}}-1\right)}\left(\varepsilon_{1} \sqrt{\beta_{\mathrm{ucf}}^{2}-\varepsilon_{2}}+\varepsilon_{2} \sqrt{\beta_{\mathrm{ucf}}^{2}-\varepsilon_{1}}\right)}{\frac{\varepsilon_{1} \varepsilon_{2}}{-\varepsilon_{\mathrm{o}}^{\mathrm{nl}}}\left(-\varepsilon_{0}^{\mathrm{nl}}\left(\frac{\beta_{\mathrm{ucf}}^{2}}{\varepsilon_{\mathrm{e}}^{\mathrm{nl}}}-1\right)\right)+\varepsilon_{\mathrm{o}}^{\mathrm{nl}}\left(\sqrt{\beta_{\mathrm{ucf}}^{2}-\varepsilon_{1}} \sqrt{\beta_{\mathrm{ucf}}^{2}-\varepsilon_{2}}\right)}\right)
\end{aligned}
$$

one can derive the following equation where integer $\mathrm{m}$ defines the number of propagating modes supported by the

$$
k_{0} h \sqrt{-\varepsilon_{0}^{\mathrm{nl}}\left(\frac{\beta_{\mathrm{ucf}}^{2}}{\varepsilon_{\mathrm{e}}^{\mathrm{nl}}}-1\right)}=m \pi-U^{\mathrm{ucf}}
$$

HMM slab, $\mathrm{m}=1,2, \ldots$ and the parameter $U^{\text {ucf }}$ would be less than $\frac{\pi}{2}$. Solving the latter equation for $m$ results in the subsequent expression

$$
m=\left[\frac{1}{\pi}\left(2 \pi N \frac{t}{\lambda} \sqrt{-\varepsilon_{0}^{\mathrm{nl}}\left(\frac{\beta_{\mathrm{ucf}}^{2}}{\varepsilon_{\mathrm{e}}^{\mathrm{nl}}}-1\right)}+U^{\mathrm{ucf}}\right)\right]_{\mathrm{int}}
$$

where $[. . .]_{\text {int }}$ stands for the first integer number greater than or equal to the value inside the brackets. Equation (15) indicates existence of finite number of modes in the finite thickness HMMs.

\subsection{Origin of LR and SR high-k modes}

Macroscopic behavior of bulk high-k modes in multilayer HMMs is well defined by the developed dispersion relations for finite HMMs. To shed light on the long-range and short-range propagating high-k modes from the microscopic point of view, one should gain insight into plasmonic phenomena behind the multilayer HMMs properties. It is shown that coupling of surface plasmon polaritons (SPPs) at metal-dielectric interfaces of the whole multilayer stacks result in bulk modes of the HMMs. [40]

SPPs on two adjacent interfaces can be coupled through either the dielectric or the metal layers. In this regard, there are two configurations for the SPPs coupling: metal-dielectric-metal (MDM) and dielectric-metal-dielectric (DMD). Surface waves coupled through the metal layer, DMD configuration, are called plasmonic slab-like modes and coupled through the dielectric layer, MDM configuration, are called plasmonic gap-like modes. [49] Figure 4a displays the DMD coupling and Figure 4b indicates the MDM coupling dispersions. Figure 4a,b also show the lossless dispersion of SPPs at a single $\mathrm{Au}-\mathrm{Al}_{2} \mathrm{O}_{3}$ interface in green dashed line. It lies below the surface plasmon frequency of metal layer that is shown with the horizontal dotted line. By coupling of SPPs, the single interface dispersion splits into two branches, below and above the single SPP ones. The red branch corresponds to symmetrically coupled and the black one corresponds to anti-symmetrically coupled SPPs. Insets of Figure 4a,b show magnetic field profiles of the symmetrically and anti-symmetrically coupled waves.

Microscopically describing bulk modes of the HMM structure with multiple metal-dielectric interfaces, one should analyze it as a composite of DMD and MDM couplings configurations. The case of considering gaplike and slab-like dispersion relations simultaneously (a simplified case happening for stacks having 2 periods), is shown in Figure 4c. One can see changes in the symmetry of fundamental mode by sweeping over $k_{\mathrm{x}}$ which corresponds to sweeping over effective index $\beta$. For lower $k_{\mathrm{x}}$ the symmetric gap-like mode appear as the fundamental mode (the branch in red), for higher $k_{\mathrm{x}}$, the fundamental mode changes to the slab-like antisymmetric mode (the branch in black). [50] However, in general, existence of such crossing region depends strongly on the layers material and thickness as well as number of periods. For stacks having low number of periods two branches may be anti-crossing. But, there would appear a transition in fundamental mode from the symmetric gap-like to the anti-symmetric slab-like modes by adding more layers and consequently widening each branch and having them crossing. As number of layer increases, each branch splits more and more that finally instead of branches there will be a band of symmetric and anti-symmetric modes (Bloch theorem). In multilayer stacks, there are always competing conditions between the slab-like and gap-like 


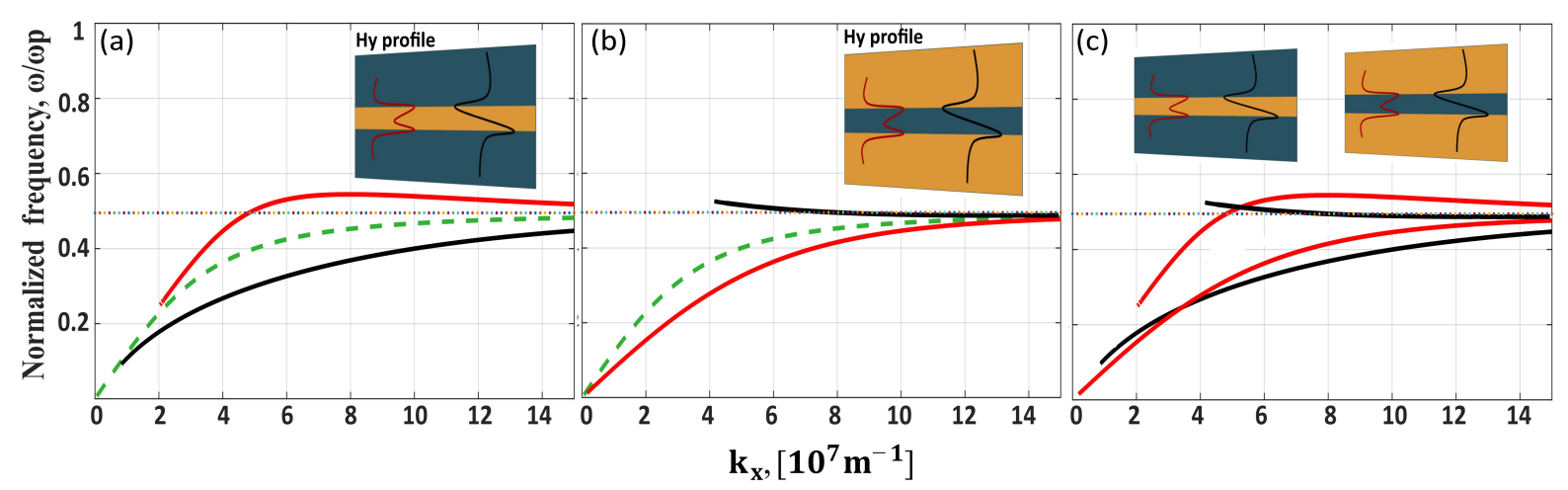

Figure 4 Dispersion of coupled surface plasmon polaritons in a) DMD, b) MDM coupling configurations. Green line shows the lossless dispersion of SPPs at the interface of $\mathrm{Au}-\mathrm{Al}_{2} \mathrm{O}_{3}$, red and black lines indicate symmetrically and anti-symmetrically coupled SPPs, respectively. Insets schematically show associated $H_{\mathrm{y}}$ mode profile for both symmetric (red) and antisymmetric (black) coupled modes. c) Combination of DMD and MDM coupled waves dispersion relations.

modes. Considering only the slab-like coupled modes with large propagation constants may mislead to reduced number of accepted high-k modes in the HMM structure. [51]

To summarize, at lower effective indices, symmetric gap-like modes prevail in coupling of SPPs on all interfaces and thus leads to the symmetric bulk high$\mathrm{k}$ waves in multilayer structures. Since the symmetrically coupled waves possess strong surface confinement and very high losses, [52] it is expected that symmetric high-k modes bear strong damping and result in short-range propagation length along the slab thickness. On the other hand, in the region of larger effective indices where anti-symmetric slab-like modes dominate, anti-symmetrically coupled SPPS show weaker surface confinement and lower loss. [52,53] Therefore, predominating anti-symmetric coupled SPPs at all interfaces brings about high-k bulk modes which behave as long-range propagating waves in the HMM slab. In the meantime, the mode number definition of the LR propagating high- $\mathrm{k}$ modes can be well explained with anti-symmetric coupling description. As the mode number corresponds to the number of zerocrossing in the $H_{\mathrm{y}}$ field profile of the corresponding mode, the anti-symmetric coupling provides multiple zeros in the field profile across the HMM slab. [51] Back to the macroscopic behaviour of bulk modes, distinctive properties of SR and LR high-k modes could be further understood. In general, LR high-k modes are weakly localized at the HMM boundaries and largely exist in the HMMs. As a result, most of the mode's energy resides in the HMM structures. A relatively long propagation length of LR modes is suitable for utilizing HMM slabs for imaging applications. Also, the low losses associated to LR modes makes them appropriate for HMMs applications for spontaneous emission enhancement. In contrast, SR high-k modes as strongly confined to the interfaces, make the energy located at the HMM interfaces and the surrounding media. Thus, subwavelength localization of the electric field in exchange of a shorter propagation length can be achieved. It should be noted that losses attributed to HMM modes are considerably lower than that of a plasmonic mode of a single metal layer. Therefore highly localized SR modes can be advantageous for waveguiding as well as sensing applications of HMMs.

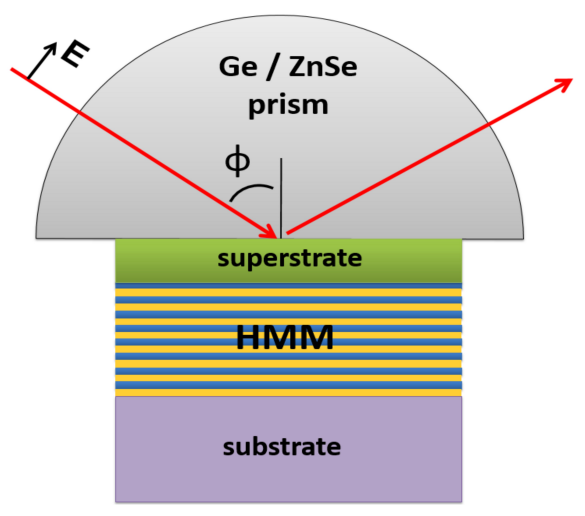

Figure 5 Otto configuration for high-k modes excitation; a high refractive-index prism is used for k-matching condition.

\section{Numerical and experimental validation}

\subsection{Transfer Matrix Method}

To examine the developed theory, high- $\mathrm{k}$ modes dispersion derived in section 3.1 are compared with the results delivered by transfer matrix method. TMM as a robust numerical approach reveals dips in the reflection spectra that signify high-k modes excitation in the multilayer structures. Using TMM, reflection spectra of multilayer HMM slabs consisting of $10 \mathrm{~nm}$ thick alternating $\mathrm{Au}$ and $\mathrm{Al}_{2} \mathrm{O}_{3}$ layers with a total thickness 


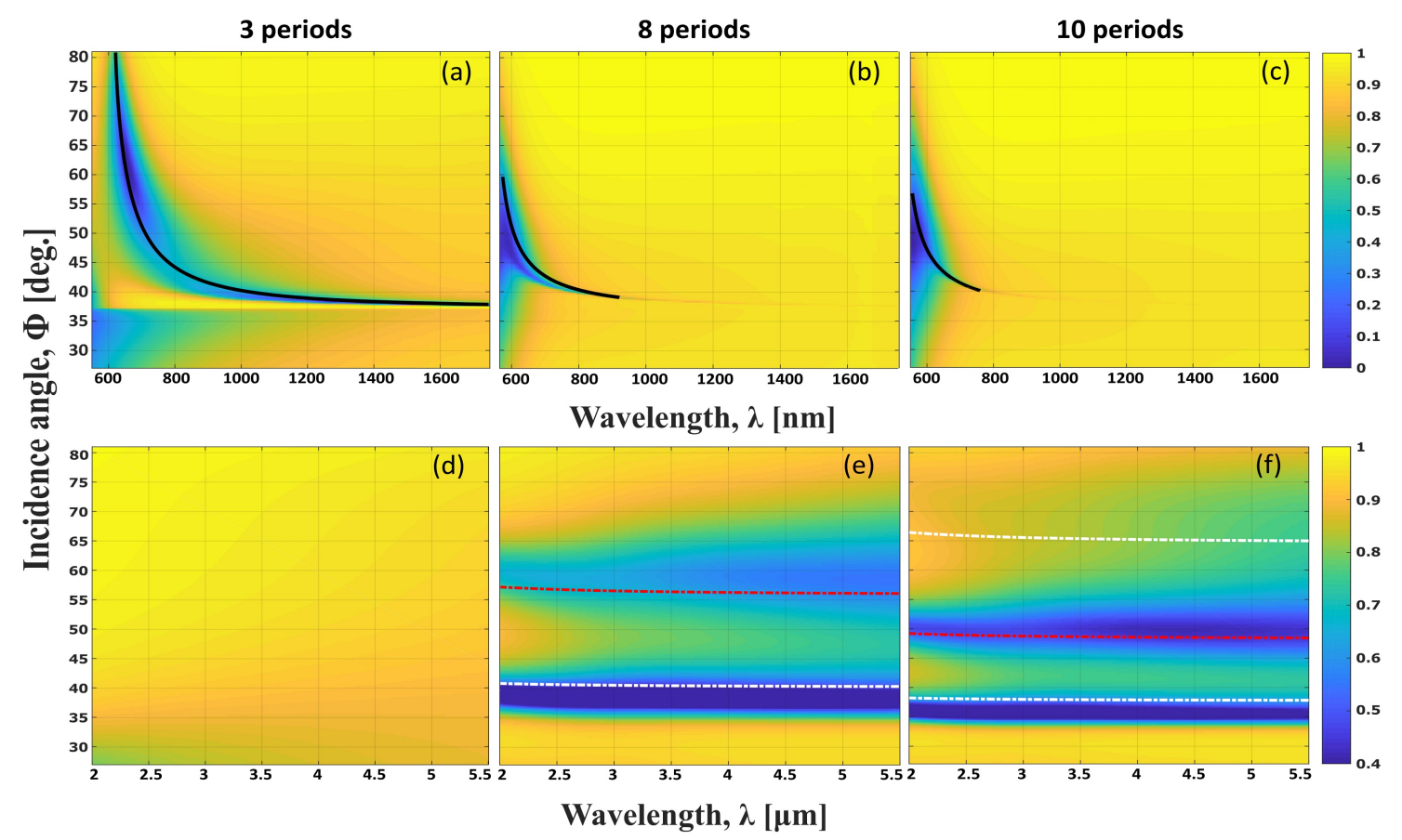

Figure 6 High-k modes dispersion in multilayer HMM slabs consisting of $10 \mathrm{~nm}$ thick $\mathrm{Au}_{-} \mathrm{Al}_{2} \mathrm{O}_{3}$ layers, with the total thickness equivalent to $(\mathrm{a}, \mathrm{d}) 3$, $(\mathrm{b}, \mathrm{e}) 8$ and $(\mathrm{c}, \mathrm{f}) 10$ periods. TMM reflection spectra shown in the background are calculated from a configuration of high-index prism - air superstrate $\left(n_{1}=1,20 \mathrm{~nm}\right)$ - Multilayer stacks - glass substrate $\left(n_{2}=1.51\right)$. In $(\mathrm{a}-\mathrm{c})$ a ZnSe prism transparent in VIS-NIR is considered. Due to the effective index provided by ZnSe prism with the range of $\beta=\left[\begin{array}{ll}1.17 & 2.46\end{array}\right]$, only SR high-k mode is excited. In (d-f) a Ge prism with higher refractive index, non-absorptive in Mid-IR, is taken to provide higher $\beta$ in the range of $\beta=$ [1.88 3.94]. High-k modes dispersion, white and red dashed lines, indicate excitation of lowest 2 and 3 LR high-k modes in HMMs having 8 and 10 periods respectively.

equal to 60,160 and $200 \mathrm{~nm}$ equivalent to 3,8 and 10 periods respectively, are calculated; where the whole stack is sandwiched between the glass substrate $\left(n_{2}=\right.$ 1.51) and air superstrate $\left(n_{1}=1\right)$. In order to provide $\mathrm{k}$-matching condition for excitation of high-k modes, we considered a high refractive-index semi-infinite material coupled with the structure resembling a prism in Otto configuration. For realistic analysis we chose a $20 \mathrm{~nm}$ thick air gap (the superstrate) between the high-index material and the multilayer stack. TM polarized light is directed through the upper layer to the structure. The system is alike to the one shown in Figure 5 .

High-k modes in visible to near-infrared wavelengths is shown in Figure 6a-c. A zinc selenide prism, $n_{\mathrm{ZnSe}} \approx 2.5$, is considered here owing to its transparency in the operating wavelengths. Implementing incidence angles in the interval $\phi=\left[\begin{array}{ll}28^{\circ} & 80^{\circ}\end{array}\right]$, the normalized transverse wavevector (effective index) lies in the interval $\beta=[1.172 .46]$; according to $\beta=n_{\mathrm{ZnSe}} \sin \phi$. High-k modes dispersion given by Eq. (9) are also shown in Figure 6a-c; the black lines correspond to short-range propagating modes. It indicates that only SR high-k modes can be excited for the provided $\beta$ and no LR modes appear. To provide higher $\beta$, a Ge prism with higher refractive index, $n_{\mathrm{Ge}} \approx 4$ non-absorptive in MidIR is considered that provides $\beta=\left[\begin{array}{ll}1.88 & 3.94\end{array}\right]$. High- $\mathrm{k}$ modes dispersion of the same structures in Mid-IR wavelengths are shown in Figure 6d-f. Lack of any excited mode in Figure 6d indicates that for HMMs having low thickness, the LR high-k modes possess high effective mode indices (as Figure 3a implies) that can not be excited commonly. On the other hand, it can be noticed from Figure 3a that the SR mode shown in black line, is not extended to higher wavelengths. Thus, for HMMs with 3 periods shown in Figure 6d, no modes can be seen. High-k modes dispersion, shown as white and red dashed lines in Figure 6e,f, point out excitation of odd and even LR modes, with use of the Ge prism, in HMM slabs having higher thicknesses. Figure $6 \mathrm{~d}$-f show very well agreement between the theoretical high-k modes dispersion and TMM reflection spectra shown in the background and authenticate the fact that increasing number of periods in multilayer stack results in shifting LR high-k modes to lower effective mode indices.

\subsection{Optical experiments}

In order to demonstrate validity of theory predictions and observe high- $\mathrm{k}$ modes, we conducted reflection measurement of the structure in Otto configuration including Ge hemispherical prism - Air gap - HMM 
slab - glass substrate as illustrated in Figure 5. The HMM structure consists of 8 periods with a unit cell of $\mathrm{Al}_{2} \mathrm{O}_{3}(10 \mathrm{~nm})-\operatorname{APTMS}(1 \mathrm{~nm})-\mathrm{Au}(10 \mathrm{~nm})-$ APTMS (1 nm). Here, APTMS stands for aminopropyltrimethoxysilane and is used as a lossless adhesion layer between $\mathrm{Au}$ and oxide layers, which is advantageous for highly localized propagating surface plasmon waves. [48] The HMM slab is fabricated on a $500 \mu \mathrm{m}-$ thick glass substrate. Each Au film is sputtered and $\mathrm{Al}_{2} \mathrm{O}_{3}$ film is deposited by atomic layer deposition. To

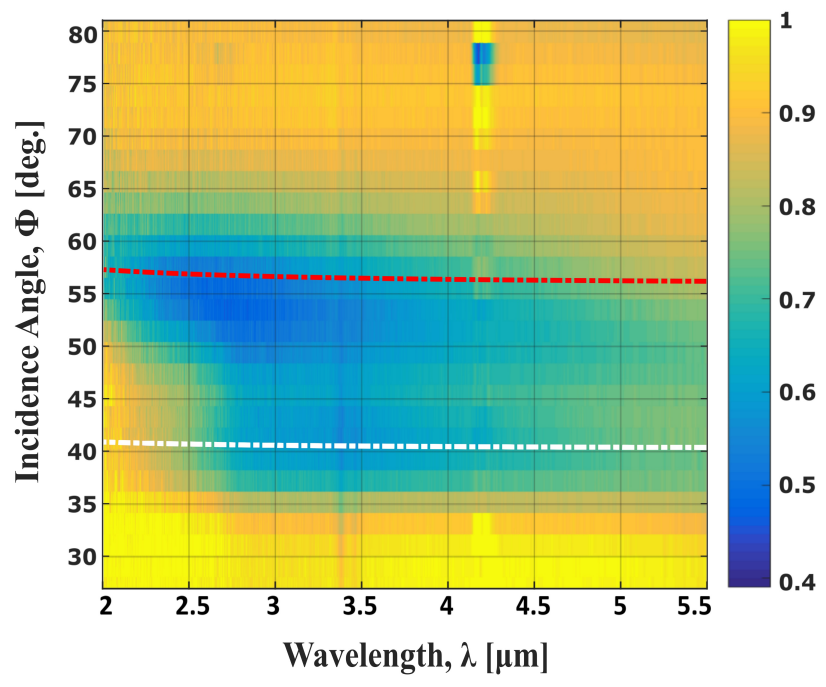

Figure 7 Experimental reflection spectrum from Ge prism Air gap $(20 \mathrm{~nm})$ - HMM slab - glass substrate. The HMM slab is made of 8 periods of $\mathrm{Al}_{2} \mathrm{O}_{3}(10 \mathrm{~nm})-\operatorname{APTMS}(1$ $\mathrm{nm})-\mathrm{Au}(10 \mathrm{~nm})-\operatorname{APTMS}(1 \mathrm{~nm})$ unit cell. Note that vertical noises around $\lambda=3.4 \mu \mathrm{m}$ and $\lambda=4.2 \mu \mathrm{m}$ are caused by the light absorption by water and $\mathrm{CO}_{2}$ in the air, respectively. The white and red dashed lines show the odd and even LR high-k modes.

excite high-k modes using a Ge prism provides us with one of the highest refractive index of $n_{\mathrm{Ge}} \approx 4.0$ in the mid-infrared wavelength range. Its transparency window $\lambda>2.0 \mu \mathrm{m}\left(5000 \mathrm{~cm}^{-1}\right)$ suits well for the midIR waves. It has been used, for example, to observe plasmons on doped semiconductors [54] and surface waves on hyperbolic metamaterials. [13] Using a hemispherical Ge prism, the incident angles are varied in the range $\phi=\left[28^{\circ} 80^{\circ}\right]$ with $2^{\circ}$ steps. This provides the larger range of effective index, $\beta=$ [1.88 3.94], extended enough to excite LR high-k modes. According to the dispersion in Figure 3e, the available $\beta$ enables us to observe not only the lowest, but also the second lowest long range propagating modes. The measurements were performed by the Fourier Transform Infrared (FTIR) spectrometer (VERTEX 70, Bruker) with the TM-polarized incident light in the wavelength range of $\lambda=2-5.5 \mu \mathrm{m}\left(5000-1882 \mathrm{~cm}^{-1}\right)$. The presented reflection spectra are averaged from 32 scans. For the reference spectrum, TM reflectance from Ge prism without HMM sample is taken at the angle of incidence $\phi=78^{\circ}$. The incident beam has angular variation of $\Delta \phi= \pm 1.7^{\circ}$. Note that the critical angle between the Ge prism and air is $14.5^{\circ}$, so the modeling and experiment were conducted well above the critical angle. Therefore, we can assume that any reflection dip should correspond to excitation of a high-k HMM mode. The thickness of the air gap is found to be $20 \mathrm{~nm}$ by fitting the experimental results with the simulated reflection spectra. The measurements show two branches of high-k modes as shown in Figure 7. White and red dashed lines illustrate theoretical predictions for the LR propagating high-k modes. The lowest mode is the odd LR and the upper one is the even LR modes. The experimental reflection shows qualitatively good agreement with the theoretical predictions for high-k modes, as well as with the TMM results. A low Q-factor of the measured reflection spectra is mainly due to the measurement uncertainties in the FTIR setup including angular beam divergence and unavoidable polarization mixture.

\section{Engineering high-k modes in HMM slabs}

\subsection{Determining effective mode index}

While propagation of waves with very large effective index is the essential property of hyperbolic metamaterials, excitation as well as out-coupling of these modes have been an important challenge in multilayer HMMs applications. High-k modes dispersion shown in Figure 3, for HMMs consisting of $\mathrm{Au}-\mathrm{Al}_{2} \mathrm{O}_{3}$ layers with 10 nm-thick, corroborate that corresponding LR modes possess such high $\beta$ that they could not be excited in visible wavelengths (see Figure $6 \mathrm{a}-\mathrm{c}$ ). Referring to Eq. (10b), the relation between $\varepsilon_{\mathrm{e}}^{\mathrm{nl}}$ and $\beta$ mainly determines the lowest possible effective mode index of LR modes. Hence, HMMs specification can be engineered to shift the LR modes to lower $\beta$ by manipulating the extraordinary permittivity component.

Equation (2b) points out dependency of $\varepsilon_{\mathrm{e}}^{\mathrm{nl}}$ on unitcell materials, $\varepsilon_{\mathrm{d}}, \varepsilon_{\mathrm{m}}$ and thickness, t as well as metal fill fraction, $\mathrm{p}$ that provide degrees of freedom to properly design HMMs. One can deduce that decreasing $\mathrm{p}$, $\mathrm{t}$ and $\varepsilon_{\mathrm{d}}$ result in a lower $\varepsilon_{\mathrm{e}}^{\mathrm{nl}}$ and consequently lower effective mode index of LR modes. To investigate the effect of reduction in $\varepsilon_{\mathrm{d}}$, we chose $\mathrm{SiO}_{2}\left(n_{\mathrm{SiO}_{2}} \approx 1.44\right)$ as an alternative dielectric material. Thus, HMM slabs consisting of $10 \mathrm{~nm}$-thick $\mathrm{Au}-\mathrm{SiO}_{2}$ alternating layers are considered between a glass substrate $\left(n_{2}=1.51\right)$ and air superstrate $\left(n_{1}=1\right)$. Figure $8 \mathrm{a}-\mathrm{c}$ show the high$\mathrm{k}$ SR (black line) and LR (blue dashed line) modes as well as TMM reflection spectra for the structures having 3, 8 and 10 periods; similar to the case in Figure $6 \mathrm{a}-\mathrm{c}$ but permittivity of dielectric layer is reduced by a factor of one, $\varepsilon_{\mathrm{Al}_{2} \mathrm{O}_{3}}-\varepsilon_{\mathrm{SiO}_{2}} \approx 1$. In comparison to Figure $6 \mathrm{a}-\mathrm{c}$, one can see excitation of the first LR mode in the HMM slab having 10 periods. It indicates that the LR effective mode index is shifted to lower values 


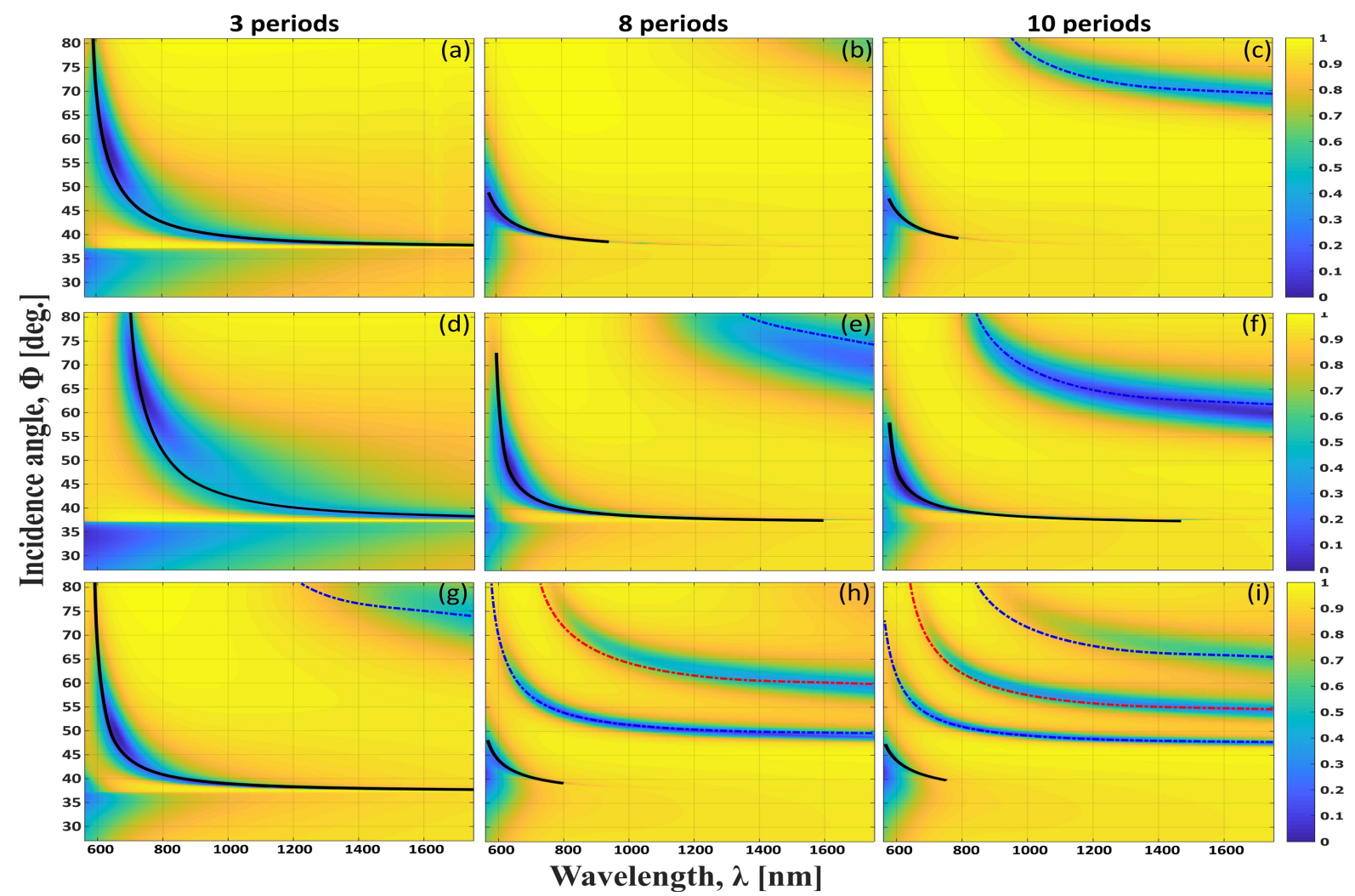

Figure 8 High-k modes of multilayer HMM slabs consisting of $\mathrm{Au}-\mathrm{SiO}_{2}$ alternating layers with (a-c) 10-10 nm, (d-f) 6.66-13.34 nm and (g-i) 15-30 nm thicks, respectively. The total thickness corresponds to (a,d) 3, (b,e) 8 and (c,f) 10 periods. Black lines show the short-range, blue and red dashed lines show the odd and even long-range high-k modes. TMM reflection spectra are shown in the backgrounds.

that for the case in Figure 6c could not be excited in the provided range of $\beta$. Subsequently, a unitcell of $\mathrm{Au}-\mathrm{SiO}_{2}$ with thickness 6.66 and $13.34 \mathrm{~nm}$ respectively, are chosen to survey the effect of decreasing fill fraction. Figure 8d-f indicate that in comparison to the results in Figure $8 \mathrm{a}-\mathrm{c}$, decreasing fill fraction from 0.5 to 0.33 leads to a decrease in effective indices of the modes. Figure $8 \mathrm{~g}$-i represent the significance of unit-cell thickness on engineering the HMMs high-k modes in order for easier excitation-extraction. As an another configuration, HMMs with unit cell of $15 \mathrm{~nm}$ $\mathrm{Au}$ and $30 \mathrm{~nm} \mathrm{SiO}_{2}$ are considered to study the effect of unit-cell thickness with a fill fraction of 0.33 similar to the case in Figure 8d-f. Figure 8g-i show high-k bulk modes dispersion for the HMM slabs with total thickness equivalent to 3,8 and 10 periods. The high-k modes realized here possess much lower effective mode indices. In other words, shifting the modes to lower $\beta$ we could excite higher number of LR modes.

Figure 8 indicates well matching between results of the developed theory and TMM as a numerical approach. And, it gives information about high degree of freedom in controlling effective mode index of LR high-k modes to be in desire range of $\beta$ by carefully designing multilayer structures.

\subsection{Number of modes}

As discussed earlier, multilayer HMMs provide finite number of long-range propagating high-k modes. With regard to importance of engineering HMM slabs for the propagating modes, we provide investigations on the potentially effective parameters. Based on Eq. (15), number of periods $\mathrm{N}$, ambient media $\varepsilon_{2}$ and $\varepsilon_{1}$, unit cell thickness t, and metal fill fraction $\mathrm{p}$, are significant involving parameters affecting number of LR modes. In what follows multilayer structures consisting of $\mathrm{Au}$ $-\mathrm{Al}_{2} \mathrm{O}_{3}$ are probed while wavelength is kept constant at $\lambda=800 \mathrm{~nm}$.

Figure 9a shows number of LR modes for HMM slabs consisting of $2-10$ periods, where $\mathrm{t}=20 \mathrm{~nm}, \mathrm{p}=$ $0.5, n_{2}=1.51$ and $n_{1}=1$. The fitted black line exposes a direct relation but not one-to-one correspondence between the number of periods and modes. Dependency of $\mathrm{m}$ on $\mathrm{N}$ looks like an affine function that neither scale multiplicatively nor necessarily preserve distances between points.

As it is clear from Eq. (13) and (15), dependency of $\mathrm{m}$ on the ambient media seems more complicated. For HMM slabs having $\mathrm{N}=3, \mathrm{p}=0.5$ and air superstrate $n_{1}=1$, Figure 9b shows inverse-nonlinear relation between $\mathrm{m}$ and substrate refractive index; wherein $n_{2}=$ 


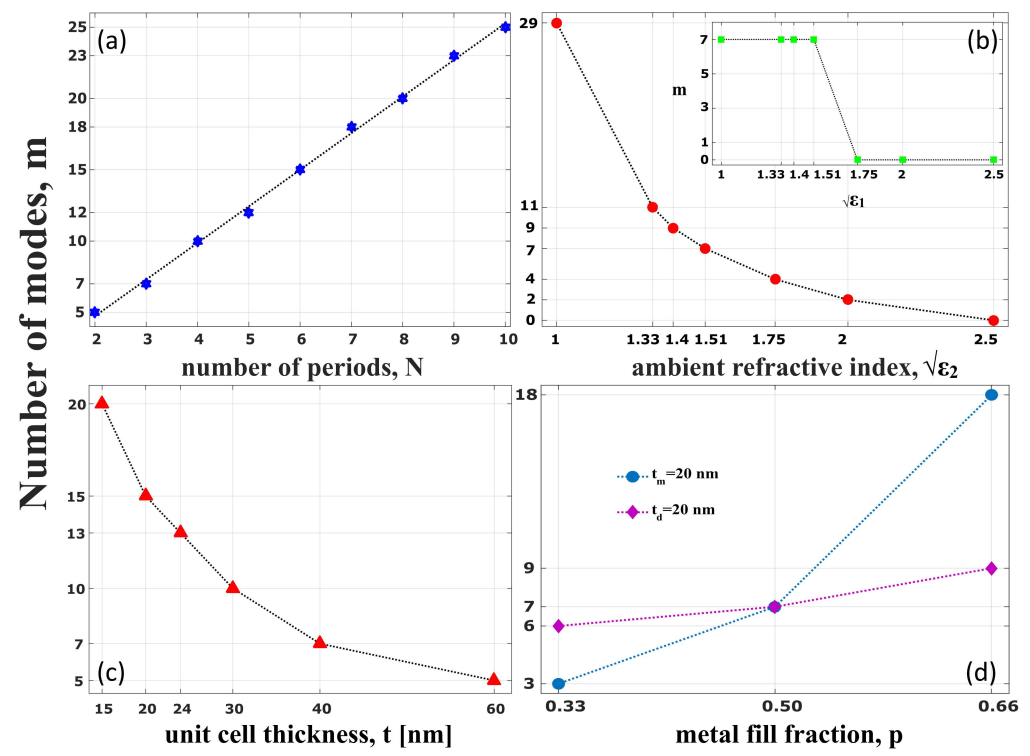

Figure 9 Dependency of high-k modes of $\mathrm{Au}-\mathrm{Al}_{2} \mathrm{O}_{3}$ HMMs at $\lambda=800 \mathrm{~nm}$ to a) number of periods, $\mathrm{N}=2-10$ ( $\mathrm{p}=$ $\left.\left.0.5, \mathrm{t}=20 \mathrm{~nm}, n_{2}=1.51, n_{1}=1\right) . \mathrm{b}\right)$ ambient media, $n_{2}=1,1.33,1.4,1.51,1.75,2$ and $2.5(\mathrm{p}=0.5, \mathrm{t}=20 \mathrm{~nm}, \mathrm{~N}=3$, $\left.n_{1}=1\right)$. The inset shows $n_{1}=1,1.33,1.4,1.51,1.75,2$ and $2.5\left(\mathrm{p}=0.5, \mathrm{t}=20 \mathrm{~nm}, \mathrm{~N}=3, n_{2}=1.51\right)$. c) thickness of unit cell, $\mathrm{t}=15,20,24,30,40$ and $60 \mathrm{~nm}$, with constant total thickness $\left(\mathrm{h}=120 \mathrm{~nm}, \mathrm{p}=0.5, n_{2}=1.51, n_{1}=1\right)$. d) metal fill fraction $\mathrm{p}=0.33,0.50$ and 0.66 . Circles indicate $t_{\mathrm{m}}=20 \mathrm{~nm}$ and $t_{\mathrm{d}}=10,20$ and $40 \mathrm{~nm}$. Diamonds represent the same fill fraction for $t_{\mathrm{d}}=20 \mathrm{~nm}$ and $t_{\mathrm{m}}=10,20$ and $40 \mathrm{~nm}$; with constant total thickness $\left(\mathrm{h}=120 \mathrm{~nm}, n_{2}=1.51, n_{1}=1\right)$.

$1,1.33,1.4,1.51,1.75,2$ and 2.5. Number of modes is trending downward as $n_{2}$ is increasing and finally $\mathrm{m}$ gets to zero as $n_{2}$ overtakes $\sqrt{\varepsilon_{\mathrm{e}}^{\mathrm{n}}}$ that violates the condition mentioned in Eq. (10b). For the same HMM slabs, the inset in Figure $9 \mathrm{~b}$ indicates almost constant dependency of $\mathrm{m}$ on $n_{1}$, while $n_{2}$ is equalled to 1.51 (glass substrate). Number of modes suddenly goes down to zero referring to the presumption that $\varepsilon_{1} \leqslant \varepsilon_{2}$. Figure $9 \mathrm{~b}$ implies that effect of denser surrounding medium is more pronounced in defining number of LR modes in addition to its main impact on the upper cut-off effective index (refer to Eq. (11) and (12)) and the direct effect on the lower cut-off.

To infer the impact of $t$ on HMMs high-k modes, Figure $9 \mathrm{c}$ presents number of LR modes vs. unit-cells thickness. Here, $\mathrm{t}=15,20,24,30,40$, and $60 \mathrm{~nm}$ while total thickness is kept constant, $\mathrm{h}=120 \mathrm{~nm}$ and $\mathrm{p}=$ $0.5, n_{2}=1.51$ and $n_{1}=1$. Inverse-nonlinear relation between $\mathrm{t}$ and $\mathrm{m}$ can be concluded; the thinner unitcell results in a higher number of modes.

Figure 9d shows investigation on high-k modes dependency on the metal fill fraction p. Keeping the total thickness of HMM slab fixed at $\mathrm{h}=120 \mathrm{~nm}$, the metaldielectric ratio of $2: 1,1: 1,1: 2$ are examined while $n_{2}$ $=1.51$ and $n_{1}=1$. Circles in Figure $9 \mathrm{~d}$ indicate the number of modes for $\mathrm{p}=0.33,0.50,0.66$ corresponding to unit cells with dielectric layer thickness $t_{\mathrm{d}}=$ 10,20 and $40 \mathrm{~nm}$ and metal layer thickness $t_{\mathrm{m}}=20$ $\mathrm{nm}$. Diamonds represent the same fill fractions for the case that the dielectric layer thickness kept constant as $t_{\mathrm{d}}=20 \mathrm{~nm}$ and metal layer thickness is changed as $t_{\mathrm{m}}=10,20$ and $40 \mathrm{~nm}$. The immediate inference would be a direct relation between metal fill fraction and number of high-k modes. Increasing $\mathrm{p}$ generally raise high-k modes in HMMs slabs. That aside, Figure $9 \mathrm{~d}$ also implies that multilayer stacks having thinner unit cell thickness provide a higher number of modes. For example, at $\mathrm{p}=0.33$ the blue circle represents a unit-cell of $\mathrm{t}=60 \mathrm{~nm}$ while the pink diamond represents $t=30 \mathrm{~nm}$. The blue dotted line implies sharp increasing number of modes from left to right because of simultaneously increasing $\mathrm{p}$ from $0.33-0.66$ and decreasing t from 60 to $30 \mathrm{~nm}$, in total accordance to the results deduced from Figure 8d-i. On the contrary, almost monotonic behaviour of pink dotted line shows competitive impact of increasing fill fraction as a positive factor and increasing unit-cell thickness as a negative factor in raising number of high-k modes.

\section{Conclusion}

The present paper soughs to address the bulk high$\mathrm{k}$ modes of empirically finite thickness multilayer hyperbolic metamaterials. In this regard, we developed a theory based on nonlocal homogenization of multilayer HMMs by applying operator approach EMA and considering waveguide rules for the finite thickness effective medium placed in semi-infinite boundary media. Distinguishing short-range and long-range propagating waves, we managed to interpret the bulk high-k modes of the metal-dielectric HMMs. The criteria for classification of the two modes and delineation for their effective mode index cut-offs is introduced. As well, a 
set of governing conditions to estimate the number of such modes for arbitrary multilayer HMM structures are properly deduced. While the TMM approach cannot unveil the nature and number of such modes, and conventional EMA itself is unable to give us a hint for proper HMM engineering towards different applications, we believe that the developed theory will assist on-purpose design of multilayer hyperbolic metamaterials with desired properties.

Acknowledgements. This work was supported by Villum Fonden "DarkSILD project" (11116) and Direktør Ib Henriksens Fond, Denmark as well as the Ministry of Science, Research and Technology of Iran. The authors would like to acknowledge the support from the Danish National Center for Micro- and Nanofabrication (DTU Danchip).

Key words: Hyperbolic metamaterials, subwavelength metaldielectric multilayers, effective medium theory, high-k modes.

\section{References}

[1] W. Cai and V. Shalaev, Optical metamaterials: Fundamentals and applications, Springer, 2010.

[2] L. Ferrari, C. Wu, D. Lepage, X. Zhang, and Z. Liu, Prog. Quantum Electron. 2015, 40, 1 .

[3] V. Drachev, V. Podolskiy, and A. Kildishev, Opt. Express 2013, 21, 15048 .

[4] Z. Liu, H. Lee, Y. Xiong, C. Sun, and X. Zhang, Science 2007, 315, 1686 .

[5] D. Lu and Z. Liu, Nat. Commun. 2012, 3, 1205 .

[6] T. Repän, A. V. Lavrinenko, and S. V. Zhukovsky, Opt. Express 2015, 23, 25350 .

[7] V. Kabashin, P. Evans, S. Pastkovsky, W. Hendren, G. Wurtz, R. Atkinson, R. Pollard, V. Podolskiy, and V. Zayats, Nat. Mater. 2009, 8, 867 .

[8] E. Shkondin, T. Repän, M. E. Aryaee Panah, A. V. Lavrinenko, and O. Takayama, ACS Appl. Nano Mater. 2018, 1, 1212 .

[9] A. V. Kildishev, A. Boltasseva, and V.M. Shalaev, Science 2013, 339, 1232009 .

[10] P. V. Kapitanova, P. Ginzburg, F. J. RodríguezFortuño, D. S. Filonov, P. M. Voroshilov, P. Belov, A. N. Poddubny, Y.S. Kivshar, G. Wurtz, and A. V. Zayats, Nat. Commun. 2014, 5, 3226 .

[11] O. Takayama, E. Shkondin, A. Bogdanov, M. E. Aryaee Pahah, K. Golenitskii, P.A. Dmitriev, T. Repän, R. Malreanu, P. Belov, F. Jensen, and A. V. Lavrinenko, ACS Photonics 2017, 4, 2899 .

[12] O. Takayama, A. Bogdanov, and A. V. Lavrinenko, J. Phys.: Condens. Matter 2017, 29, 463001.

[13] O. Takayama, P. Dmitriev, E. Shkondin, O. Yermakov, M. E. A. Panah, K. Golenitskii, F. Jensen, A. Bogdanov, and A. V. Lavrinenko, Semiconductors 2018, 52, 442 .

[14] Z. Jacob, J. Y. Kim, G. V. Naik, A. Boltasseva, E. E. Narimanov, and V. M. Shalaev, Appl. Phys. B: Lasers Opt. 2010, 100, 215.
[15] J. Kim, V.P. Drachev, Z. Jacob, G. V. Naik, a. Boltasseva, E. E. Narimanov, and V.M. Shalaev, Opt. Express 2012, 20, 8100 .

[16] M. Y. Shalaginov, S. Ishii, J. Liu, J. Liu, J. Irudayaraj, A. Lagutchev, A. V. Kildishev, and V. M. Shalaev, Appl. Phys. Lett. 2013, 102, 173114.

[17] D. Lu, J. J. Kan, E. E. Fullerton, and Z. Liu, Nat. Nanotechnol. 2014, 9, 48 .

[18] M. Y. Shalaginov, V. V. Vorobyov, J. Liu, M. Ferrera, A. V. Akimov, A. Lagutchev, A. N. Smolyaninov, V. V. Klimov, J. Irudayaraj, A. V. Kildishev, A. Boltasseva, and V. M. Shalaev, Laser Photonics Rev. 2015, 9, 120 .

[19] L. Ferrari, D. Lu, D. Lepage, and Z. Liu, Opt. Express 2014, 22, 4301 .

[20] S. Ishii, A. V. Kildishev, E. Narimanov, V. M. Shalaev, and V.P. Drachev, Laser Photonics Rev. 2013, 7,265 .

[21] H. N.S. Krishnamoorthy, Z. Jacob, E. Narimanov, I. Kretzschmar, and V. M. Menon, Science 2012, 336, 205

[22] M. A. Noginov, Y. A. Barnakov, G. Zhu, T. Tumkur, H. Li, and E. E. Narimanov, Appl. Phys. Lett. 2009, 94, 151105 .

[23] S. V. Zhukovsky, A. Andryieuski, J. E. Sipe, and A. Lavrinenko, Phys. Rev. B 2014, 90, 155429.

[24] T. C. CHOY, Effective Medium Theory: Principles and Applications, 2nd ed., Oxford Science Publications, 2016.

[25] D. R. Smith, D. C. Vier, T. Koschny, and C. M. Soukoulis, Phys. Rev. E 2005, 71, 036617.

[26] T. Galfsky, E. Narimanov, and V. Menon, FiO 2015 , FW6A.3

[27] C. L. Cortes, W. Newman, S. Molesky, and Z. Jacob, J. Opt. 2012, 14, 063001 .

[28] P. Shekhar, J. Atkinson, and Z. Jacob, Nano Convergence 2014, 1, 14 .

[29] T. Galfsky, H. N. S. Krishnamoorthy, W. Newman, E. E. Narimanov, Z. Jacob, and V. M. Menon, Optica 2015, 2, 62 .

[30] P. Yeh, Optical Waves in Layered Media, Wiley Series, 1988.

[31] P. Yeh, A. Yariv, and C.S. Hong, JOSA 1977, 67, 423 .

[32] J. Elser, V.A. Podolskiy, I. Salakhutdinov, and I. Avrutsky, Appl. Phys. Lett. 2007, 90, 191109 .

[33] R. L. Chern, Opt. Express 2013, 21, 16514.

[34] A. V. Chebykin, A. A. Orlov, A. V. Vozianova, S. I. Maslovski, Y.S. Kivshar, and P. A. Belov, Phys. Rev. B 2011, 84, 115438 .

[35] X. Lei, L. Mao, Y. Lu, and P. Wang, Phys. Rev. B 2017, 96, 035439 .

[36] A. Poddubny, I. Iorsh, P. Belov, and Y. Kivshar, Nat. Photonics 2013, 7, 948 .

[37] A. Goncharenko, Y. C. Chang, R. J. Knize, and A. O. Pinchuk, Opt. Mater. Express 2017, 7, 766 .

[38] A. A. Orlov, P. M. Voroshilov, P. A. Belov, and Y.S. Kivshar, Phys. Rev. B 2011, 84, 045424.

[39] V. Popov, A. V. Lavrinenko, and A. Novitsky, Phys. Rev. B 2016, 94, 085428.

[40] S. V. Zhukovsky, O. Kidwai, and J. E. Sipe, Opt. Express 2013, 21, 14982 . 
[41] V. Popov, A. V. Lavrinenko, and A. Novitsky, Phys. Rev. B 2018, 97, 125428.

[42] J. Benedicto, R. Pollès, C. Ciracì, E. Centeno, D. R. Smith, and A. Moreau, J. Opt. Soc. Am. A 2015, 32, 1581 .

[43] V.E. Babicheva, J. Opt. 2017, 19, 124013.

[44] S. Liang, C. Jiang, Z. Yang, D. Li, W. Zhang, T. Mei, and D. Zhang, J. Opt. 2018, 20, 065001.

[45] V. E. Babicheva, M. Y. Shalaginov, S. Ishii, A. Boltasseva, and A. V. Kildishev, Opt. Express 2015, 23, 31109 .

[46] V.E. Babicheva, M. Y. Shalaginov, S. Ishii, and A. V. Kildishev, Opt. Express 2015, 23, 9681.

[47] B. E. A. Saleh and M. C. Teich, Fundamentals of Photonics, 2nd ed., Wiley Series, 2007.

[48] J. Sukham, O. Takayama, A. Lavrinenko, and R. Malureanu, ACS Appl. Mater. Interfaces 2017, 9, 25049 .

[49] J. A. Dionne, E. Verhagen, A. Polman, and H. A. Atwater, Opt. Express 2008, 16, 19001.

[50] G. Rosenblatt and M. Orenstein, Opt. Express 2011, 19, 20372 .

[51] M. Higuchi and J. Takahara, Opt. Express 2018, 26, 1918 .

[52] Z. Han and S. I. Bozhevolnyi, Rep. Prog. Phys. 2013, 76, 016402 .

[53] P. Berini, Adv. Opt. Photonics 2009, 1, 484.

[54] M. E. A. Panah, O. Takayama, S. V. Morozov, K. E. Kudryavtsev, E. S. Semenova, and A. V. Lavrinenko, Opt. Express 2016, 24, 29077 . 